\title{
CHRISTIAN HUMANISM AND
}

THE REFORMATION 



\title{
CHRISTIAN HUMANISM AND THE REFORMATION
}

\section{SELECTED WRITINGS OF ERASMUS}

WITH HIS LIFE BY BEATUS RHENANUS AND A BIOGRAPHICAL SKETCH BY THE EDITOR

\author{
Edited by \\ JoHN C. OLIN
}

Third Edition

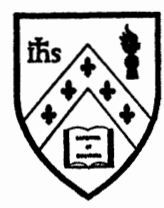

FORDHAM UNIVERSITY PRESS

NEW YORK

1987 


\section{(C) Copyright 1965 by John C. Olin \\ (C) Copyright 1975, 1979 by Fordham University Press \\ (C) Copyright 1987 by FORDHAM UNIVERSITY \\ All rights reserved. \\ LC $65-10218$ \\ ISBN $0-8232-1192-4$}

First edition 1965

Second edition 1975

Third edition 1987

Printed in the United States of America 